\title{
Sensibilisation au développement d'accélérateurs matériels sur FPGA
}

\author{
Bertrand LE GAL, Dominique DALLET \\ Institut Polytechnique de Bordeaux (IPB) \\ Composante ENSEIRB-MATMECA, Département Electronique \\ 1, avenue du Dr Albert Schweitzer - BP 99 - 33402 TALENCE Cedex - France \\ e-mail : bertrand.legal@ipb.fr et dominique.dallet@ipb.fr
}

\section{Résumé}

Les applications liées au traitement du signal et à la vidéo sont propices pour la mise en exergue des besoins d'implantation de certaines fonctions sous forme de blocs matériels dédiés. Cette implantation matérielle est une tache complexe qui nécessite des connaissances approfondies dans domaines des circuits ciblés et des méthodologies de conception. En effet, les choix réalisés au niveau algorithmiques ont des répercussions importantes sur les performances des circuits à concevoir. Partant de ce constat, nous avons décidé de créer une séquence pédagogique ciblant des étudiants de $3^{\text {ème }}$ année (master 2, semestre 9) pour les sensibiliser à cette problématique. La réalisation de cet enseignement passe par trois étapes principales. Dans un premier temps, nous fournissons aux étudiants les codes VHDL réalisant les opérations arithmétiques usuelles afin de mettre en évidence les performances que peuvent atteindre les circuits en fonction du format des données. Dans un second temps, les étudiants doivent concevoir un accélérateur matériel (pour la norme JPEG-2000) sous différentes formes, démontrant ainsi l'impact du choix de l'architecture sur les performances. Enfin dans un dernier temps, nous relions l'accélérateur matériel à un processeur SoftCore dans le FPGA afin de (1) valider fonctionnellement le circuit développé; (2) comparer les performances à celles obtenues dans une approche purement logicielle.

L'objectif de cet article est de présenter le travail réalisé par les étudiants ainsi que les objectifs pédagogiques atteints. L'ensemble des documents nécessaires à la séquence pédagogique ainsi que les codes sources sont librement accessibles sur internet. Cette démarche vise à permettre une transposition de l'enseignement au sein d'autres structures manifestant les mêmes besoins.

\section{Mots clés}

Adéquation Algorithme Architecture, Conception de systèmes numériques, Opérateurs Arithmétiques, FPGA, Traitement du Signal et de l'Image. 


\section{Introduction}

La conception de systèmes numériques embarqués s'appuie très largement sur l'utilisation d'accélérateurs matériels en supplément de processeurs généralistes ou dédiés. L'utilisation de circuits dédiés s'est accentuée durant la dernière décennie, et cela tout particulièrement dans les produits multimédias : téléphones portables, consoles de jeux, lecteurs audio et vidéo, etc. Leur présence dans de nombreux produits s'explique par l'augmentation de la complexité des applications, couplée au besoin de respecter des contraintes temps réel. Pour répondre au challenge que constitue leur intégration, les méthodes de conception ont évoluées. De plus, la définition des applications au niveau algorithmique doit prendre en considération des problématiques d'intégration afin d'assurer une adéquation algorithme architecture efficace.

La compréhension ou la définition d'applications de traitement du signal et de l'image (TSI), ainsi que leur mise en œuvre pour un étudiant ingénieur en filière électronique n'est pas simple. En effet, ces étapes nécessitent des connaissances transverses dans de multiples domaines. Lors de l'implantation d'algorithmes pour des applications TSI, il est nécessaire que les problèmes d'intégration soient pris en considération dès les phases de spécification. Pour cela, il est indispensable que, durant la formation des étudiants ingénieurs, une sensibilisation sur ce point soit menée.

C'est ce que nous nous proposons de faire à travers cet enchainement de travaux pratiques qui concernent l'étude de l'implantation d'un filtre passe-haut issu de la norme JPEG-2000 [Lawsonand 2002] [Jin 2003]. Cette étude démarre par l'analyse des performances des opérateurs de calcul élémentaires (entiers) qui seront utilisés. Ensuite, elle se poursuit par l'évaluation des opérateurs flottants (norme IEEE-754) couramment employés dans des logiciels tel MATLAB lors de la mise au point d'algorithmes. Puis, elle présente différentes solutions architecturales permettant l'implantation d'un filtre de type RIF. Enfin, elle se termine par l'intégration conjointe de l'accélérateur matériel couplé avec un processeur SoftCore et une comparaison de performances vis-à-vis d'un processeur de type Pentium.

Pour que les étudiants mènent à bien cette série de travaux pratiques, nous proposons une approche didactique. Les étudiants, en binôme, progressent par étape dans la compréhension des mécanismes de décisions permettant d'aboutir à des solutions matérielles efficaces tout en évaluant l'impact des contraintes liées aux algorithmes. À travers ces séances de travaux pratiques nous souhaitons développer les compétences d'autonomie, de réflexion et de curiosité intellectuelle, pour préparer au mieux aux attentes les étudiants à leurs futures fonctions.

Après avoir présenté la formation à l'IPB ENSEIRB-MATMECA et plus particulièrement la spécialité Traitement du Signal et de l'Image (TSI), nous réaliserons une présentation de la séquence pédagogique. Celle-ci nous permettra de montrer sa finalité vis-à-vis de la formation des élèves ingénieurs. Puis une présentation technique des travaux pratiques viendra donner les éléments 
indispensables à la compréhension de leurs mises en œuvre. Nous exposerons aussi dans cette partie les principaux résultats obtenus par les étudiants.

\section{L'option TSI (Traitement du Signal et de l'Image) du département électronique de l'IPB ENSEIRB-MATMECA}

Née en 1920 au sein de la Faculté des Sciences de Bordeaux, l'ENSEIRB (École Nationale Supérieure d'Électronique, Informatique et Radiocommunications de Bordeaux) est une des plus anciennes écoles d'ingénieurs dans le domaine de l'électronique. Elle est habilitée par la Commission des Titres d'Ingénieurs (CTI) et depuis 1975 elle fait parti du réseau des ENSI (Écoles Nationales Supérieures d'Ingénieurs). Elle recrute principalement ses effectifs sur les Concours Communs Polytechniques. Les effectifs de l'école, environ 800 étudiants sur les trois années de formation, se répartissent dans six départements d'enseignement: électronique, informatique, télécommunications, mathématique et mécanique, réseaux et systèmes d'information et système électroniques embarqués (les deux dernières sont des filières de formation d'ingénieurs par l'apprentissage). Depuis 2009, elle fait partie d'un regroupement appelé IPB (Institut Polytechnique de Bordeaux).

Le projet pédagogique de l'ENSEIRB-MATMECA est de former des ingénieurs aux compétences multiples et opérationnelles, aptes à s'épanouir dans leur vie professionnelle et à devenir des éléments moteurs du développement de leur entreprise. L'ENSEIRB-MATMECA s'efforce ainsi de concilier, dans ses objectifs de formation, le développement des deux qualités fondamentales de l'ingénieur d'aujourd'hui : l'efficacité immédiate pour les entreprises et l'indispensable adaptabilité aux mutations technologiques. Dans ces conditions, exerçant dans un secteur qui privilégie l'innovation et implique par conséquent une évolution permanente, l'ENSEIRB- MATMECA inculque à ses élèves une vaste culture pluridisciplinaire associée à une connaissance approfondie des techniques de pointe.

\subsection{Organisation à l'ENSEIRB-MATMECA : les six départements de formation}

Le département électronique offre une formation couvrant les principaux domaines de l'électronique, de l'analogique au numérique, auxquels on peut ajouter l'apprentissage des approches systèmes, indispensables pour les nouvelles technologies. Les principaux secteurs visés par cette formation sont la micro-électronique, les équipements de télécommunications, les équipements informatiques et multimédias, l'aéronautique, l'automobile et l'instrumentation.

Le département informatique propose une formation qui couvre tous les domaines de l'informatique, aussi bien dans les aspects théoriques et fondamentaux, que dans une mise en pratique effective sur ordinateur. Cette formation vise les principaux secteurs de l'ingénierie informatique comme l'analyse, la programmation, les systèmes d'informations et les réseaux.

Le département télécommunications forme des ingénieurs intégrateurs de systèmes de télécommunications. Leur savoir-faire repose sur les compétences suivantes: conception et 
développement des équipements matériels et des logiciels pour les télécommunications et les réseaux, maîtrise des systèmes adaptés aux télécommunications et aux réseaux, maîtrise des architectures et des applications réparties.

Le département mathématique et mécanique («MATMECA ») forme des ingénieurs maîtrisant les grands outils de simulation numérique et informatique. Leur savoir-faire repose sur les compétences suivantes: compréhension des phénomènes physiques et mécaniques (mécanique des solides et des structures, mécanique des fluides, ondes et vibrations), analyse et développement de modèles numériques, conception d'outils informatiques nécessaires à ce type d'étude.

Le département réseaux et systèmes d'informations forme par alternance des ingénieurs qui pourront intervenir à tous les niveaux du système d'information de l'entreprise, à savoir : les domaines techniques que recouvrent les applications d'informatique générale et industrielle, les domaines fonctionnels liés à l'analyse des organisations existantes et la maîtrise des interactions entre les différents processus « métiers ».

Le département systèmes électroniques embarqués forme par alternance des ingénieurs qui pourront intervenir à tous les niveaux de la conception des systèmes électroniques embarqués (principalement numériques), à savoir: les domaines techniques que recouvrent les systèmes sur FPGA, ASIC et l'informatique embarqué.

Toutes les formations sont semestrialisées, et la durée de la formation théorique-pratique à l'école est de cinq semestres. Le dernier semestre de la formation est quant à lui consacré à la formation pratique en entreprise sauf dans le cas de l'alternance. En effet, durant ce semestre l'élève ingénieur réalise son projet de fin d'étude dans un cadre industriel. La dernière année de formation se fait donc à moitié à l'école et à moitié en entreprise. Durant cette dernière année, au cinquième semestre de sa formation, l'élève ingénieur choisi une spécialité. Par exemple, au département électronique il peut choisir une des options de spécialisation parmi les suivantes : Traitement du Signal et des Images, Systèmes de Radio Télécommunications, Systèmes Embarqués, Circuits et Systèmes Intégrés, Automatique Mécatronique Aéronautique Automobile et Spatial. L'élève peut également choisir une option de spécialisation transversale à tous les départements de l'école (hors département par alternance) parmi les suivantes: E-Management, Ingénierie du Risque Économique, Sûreté de Fonctionnement, Technologies Multimédia.

Le projet d'enseignement que nous proposons dans cet article rentre dans la formation proposée en dernière année aux élèves du département électronique choisissant l'option Traitement du Signal et de l'Image que nous présentons dans la section suivante.

\subsection{Présentation de l'option TSI}

L'objectif de la formation proposée aux élèves du département électronique de l'ENSIERBMATMECA qui choisissent cette spécialisation de dernière année est de leur apporter une 
connaissance théorique et approfondie des techniques du traitement du signal et plus particulièrement de l'image.

Leurs compétences sont étendues à l'implantation des techniques de traitement vidéo sur cibles logicielles généralistes et dédiées (DSP et GPU) ainsi qu'aux cibles matérielles (FPGA). Ainsi, les élèves disposent en fin de formation de compétences en: traitement du signal et de l'image, électronique (analogique et numérique) ainsi qu'en informatique industrielle.

Durant le semestre de spécialisation TSI, les étudiants reçoivent de nombreux enseignements théoriques et pratiques du domaine concerné, comme : les techniques avancées de transformation et d'analyse d'images, les standards de compression vidéo, les techniques avancées de programmation en C, les architectures de processeur spécialisées pour le TSI, l'utilisation de GPU pour le calcul scientifique, ainsi que de nombreux enseignements plus larges de culture de l'ingénieur et de langues vivantes.

\section{Introduction au besoin de transdisciplinarité}

Dans le cadre de cet enseignement, nous avons souhaité mettre en place un ensemble de travaux pratiques afin de permettre aux étudiants d'utiliser les connaissances acquises durant les enseignements théoriques.

Pour réaliser un tel enseignement, nous nous sommes basé sur la séquence pédagogique de l'enseignement des matières en lien avec le numérique (et la conception matérielle) à l'ENSEIRBMATMECA. La figure suivante présente les enseignements connexes ainsi que leur chronologie.

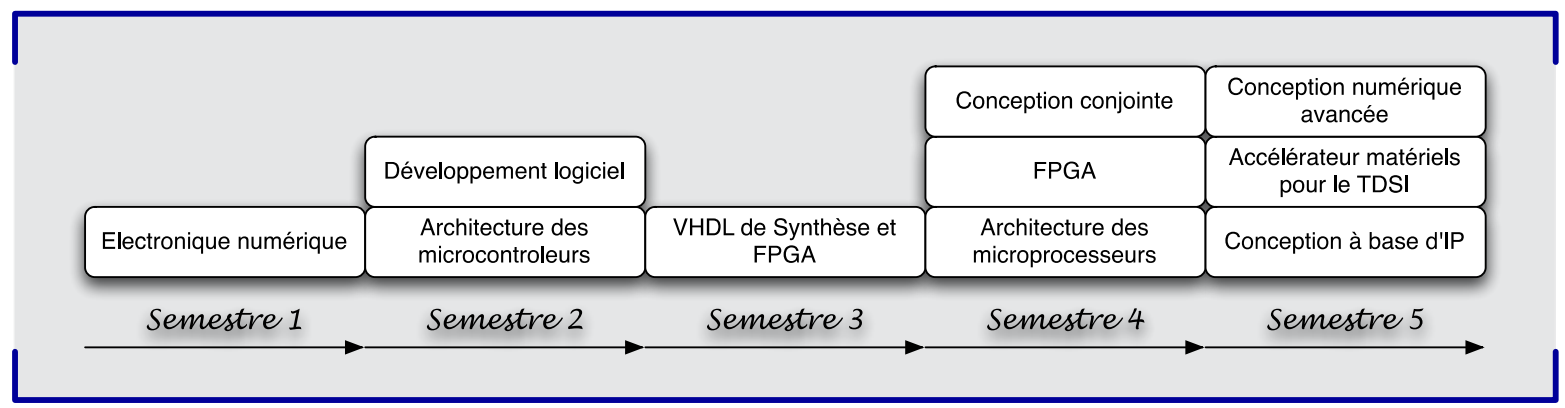

Figure 1 : présentation de l'enseignement du numérique en filière électronique option TSI

L'étude de l'implantation numérique sur CPLD et FPGA est abordée durant les 2 premières années du cursus. Ces enseignements sont prodigués de manière très généraliste et visent à fournir les bases minimum pour l'implantation de systèmes numériques sur cibles matérielles (de type FPGA). Ces enseignements sont réalisés en parallèle des cours de conception logicielle et d'architecture des ordinateurs.

Dans les enseignements prodigués en dernière année se trouvent bon nombre de cours sur la création d'algorithmes de traitement du signal à l'aide de l'environnement MATLAB. Ces enseignements 
adressent des problématiques théoriques. Une initiation au C/ASM pour le traitement du signal est réalisée sur processeur généraliste, sur DSP (processeurs spécialisés) et processeurs graphiques (GPU). Toutefois, aucune initiation n'était proposée aux étudiants sur la conception matérielle pour les systèmes TSI (conception matérielle ou conception conjointe logicielle et matérielle). Ce dernier point est désormais traité à l'aide de la séquence pédagogique présentée dans ce document.

\section{Présentation de la séquence pédagogique}

Le but pédagogique de ce projet est de développer les capacités d'analyse et d'autonomie des étudiants en leur proposant de concevoir un accélérateur matériel sur FPGA.

Dans le cadre de ce projet, nous nous adressons à des étudiants en fin de formation, c'est pourquoi l'encadrement durant les séances de travaux pratiques est le plus léger possible : les phases d'analyse et de justification des résultats sont laissées à leur charge et notées en fin de séquence. Dans un premier temps, les étudiants expérimentent différentes implantations matérielles d'opérateurs arithmétiques, qui par expériences successives leur donnent une vue d'ensemble de l'évolution des performances en fonction du type d'opération et du format des données. Ces résultats les conduisent rapidement à analyser les causes d'une telle évolution, concluant ensuite sur l'intérêt des différentes approches. C'est en se rapprochant d'une application réelle, comme nous le faisons dans la suite des TPs, que les étudiants se rendent compte de l'impact global de leurs décisions au niveau algorithmique sur les performances du système matériel à concevoir. Enfin, la validation des performances sur FPGA prouve la véracité des conclusions issues de l'analyse théorique.

Les étudiants en binôme doivent réaliser l'ensemble des travaux pratiques dans un volume horaire de 16 heures encadrées. Les salles de travaux pratiques étant en libre accès, les étudiants peuvent travailler en dehors des heures planifiées. Bien que regroupés en binôme, nous encourageons les étudiants à échanger des informations et à réfléchir en équipe afin d'affiner leurs analyses et leurs conclusions face aux phénomènes observés.

L'évaluation du projet prend en compte plusieurs points : l'attitude durant les séances, son autonomie, un rapport écrit par binôme présentant les principaux résultats expérimentaux, l'analyse des phénomènes observés ainsi qu'une conclusion sur l'impact du TSI sur les architectures matérielles.

\section{Les objectifs pédagogiques}

À travers la série de travaux pratiques présentés dans l'article, plusieurs objectifs pédagogiques distincts sont ciblés:

- Comprendre l'évolution des performances des opérateurs arithmétiques sur FPGA en fonction : du type de codage utilisé par les opérandes (entier et flottant) et de la taille des données. 
- Implanter matériellement un filtre usuel en TSI (filtre de type RIF) et évaluer l'impact du choix de l'architecture du filtre (combinatoire, pipeline, séquentielle) sur les performances (débit, surface).

- Relativiser l'impact de la fréquence de fonctionnement (faible sur FPGA) vis-à-vis des performances obtenues sur un processeur généraliste possédant une fréquence d'horloge plus élevée.

- Mettre en œuvre l'accélérateur matériel dans un flot de conception conjointe, le valider fonctionnellement sur carte et mesurer le gain en performance.

\section{Étape 1 : Implémentation des opérateurs arithmétiques entiers}

L'objectif du premier TP est de permettre aux étudiants de mesurer et d'analyser les performances obtenues sur FPGA lors de l'implantation d'opérateurs arithmétiques élémentaires. Cette première étape permet de mettre en exergue les différentes solutions dont dispose l'outil de synthèse logique lors de la réalisation de ces opérateurs arithmétiques. Elle met aussi en évidence l'évolution de leurs caractéristiques en fonction de la dynamique des données manipulées.

\section{Partie 1 : synthèse d'opérateurs arithmétiques élémentaires}

La première série d'expériences vise à intégrer des opérateurs arithmétiques (addition et multiplication) manipulant des données entières. Les opérateurs arithmétiques sont implantés dans un premier temps de manière combinatoire uniquement (l'outil utilise exclusivement des Look-Up Tables pour leurs implantations). Les mesures de performances effectuées pour des tailles d'opérandes variant de 4 à 64 bits (Figure 2) démontrent aux étudiants que :

- L'augmentation du coût en surface (Figure 2a) est linéaire vis à vis de la taille des données pour l'addition alors que pour la multiplication, l'augmentation est polynômiale. Cette différence s'explique par l'architecture interne des opérateurs : la multiplication se décompose sous forme d'additions successives des produits partiels [Ercegovac 2003] [Tisserand 2004] .

- L'allongement du chemin critique (Figure 2b) dans les opérateurs arithmétique dépend lui aussi de la nature de l'opération implantée. Toutefois, son évolution est dans les deux cas une progression quasi-linéaire. 

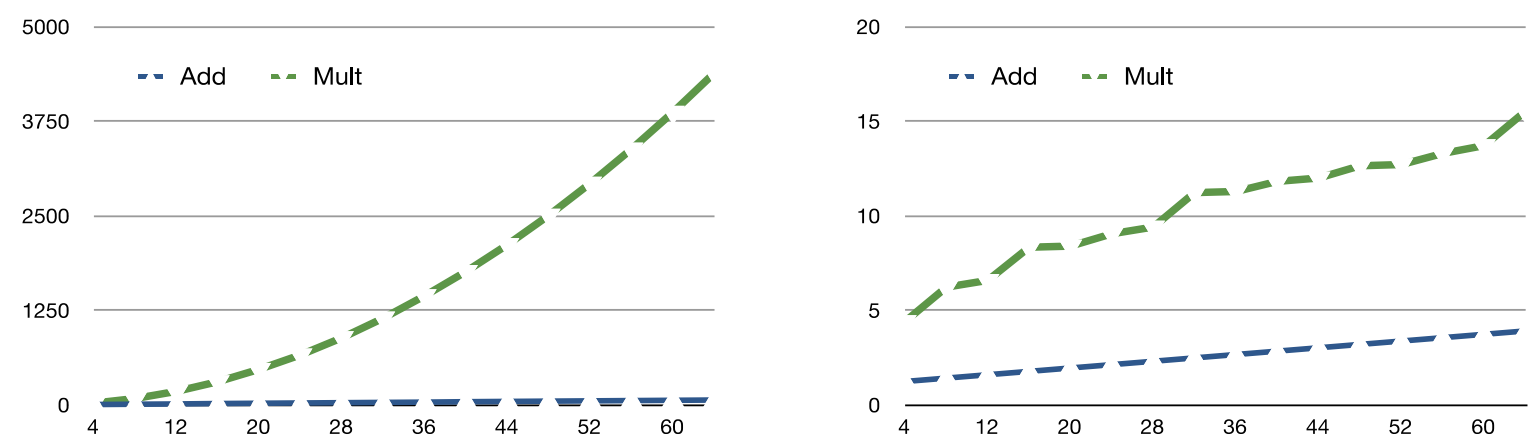

Figure 2 : Évolution de caractéristiques des opérateurs (Add/Mult) entiers sur FPGA

(a) Occupation en nombre de LUTs (b) Évolution du chemin critique en nanosecondes

On constate que les performances diminuent toujours lorsque le nombre de bits à traiter augmente. A travers ce constant assez intuitif, il est montré aux étudiants une différence fondamentale entre la conception logicielle et la conception matérielle sur FPGA : des performances sont différentes en fonction du nombre de bits utilisé (surface, débit, latence, consommation d'énergie, etc.).

\section{Partie 2 : utilisation des blocs matériels dédiés}

Les différentes familles de FPGA actuelles possèdent des ressources précablées. Ces ressources ont pour intérêt d'améliorer l'implantation des opérateurs arithmétiques vis-à-vis des implantations en LUTs (latence, débit). Le FPGA Virtex-4 [Xilinx 2008a] employé dans cette séquence pédagogique possède des blocs DSP 48 bits [Xilinx 2008b] dédiés aux besoins des applications de traitement du signal. Ces ressources dédiées sont soit automatiquement utilisées par le synthétiseur logique afin d'améliorer les caractéristiques du circuit, soit sélectionnées manuellement par le concepteur.

La seconde partie du travail demandée aux étudiants consiste à évaluer l'impact de telles ressources lors de l'implantation de multiplications. L'estimation des performances est effectuée en forçant l'outil de synthèse logique à utiliser ces ressources. Les performances obtenues en termes de "surface » utilisée et de délai du chemin critique sont fournies dans la figure 3. Il est important de noter, l'utilisation de blocs DSP nécessite dans certains cas l'utilisation de LUTs (communication entre les DSP blocs et réalisation de calculs temporaires).
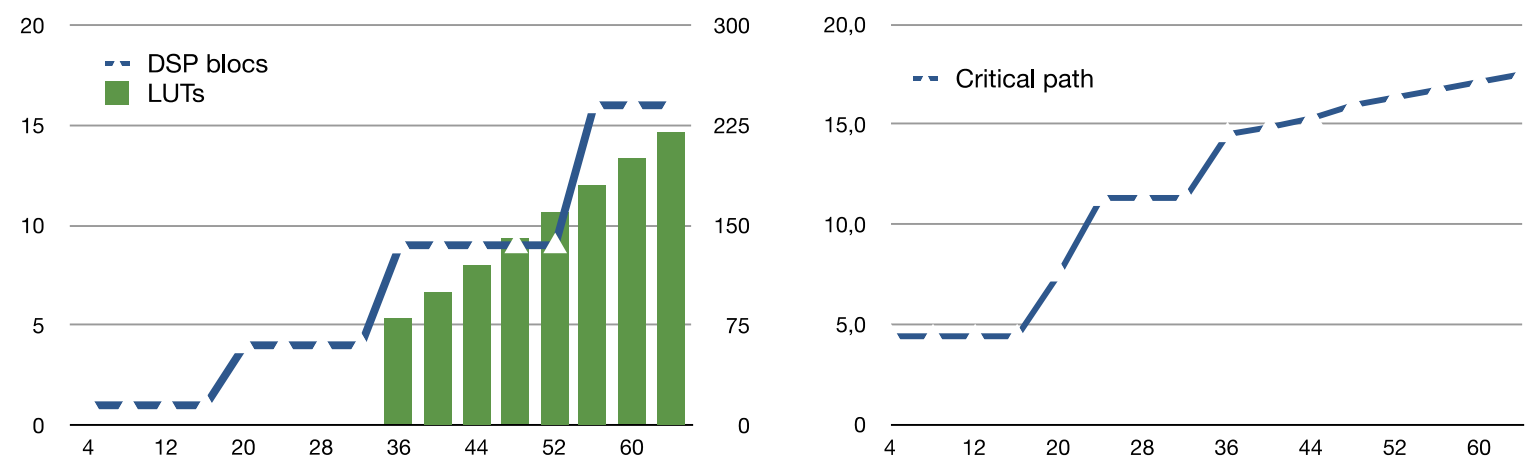

Figure 3 : Évolution des caractéristiques des opérateurs de multiplication entiers implantés sur FPGA à l'aide de blocs DSP (a) Occupation en surface (b) Évolution du chemin critique en nanosecondes 
Le nombre de blocs DSP (Figure 3a) utilisé varie en fonction de la taille des données. En effet, les DSP-48 bits sont limités par la taille des entrées, figée à 18 bits. Cette caractéristique explique l'évolution de leur nombre sous forme de palier en fonction de la taille des opérandes. Cette évolution se retrouve aussi dans l'évolution du chemin critique (Figure $3 b$ ).

La comparaison (Figure 4) entre l'implantation de l'opération de multiplication sous forme de LUTs et sous forme de blocs DSP prouve que ces blocs «optimisés » sont plus performants (chemin critique plus court) lorsque les opérandes ne dépassent pas la taille des entrées du bloc DSP. Dans le cas contraire, la sérialisation des blocs DSP dégrade les performances et peut fournir des résultats moins intéressants. Ce constat est dû à la répartition des blocs DSP dans le FPGA qui nécessite des délais de routage importants vis à vis des gains obtenus en interne.

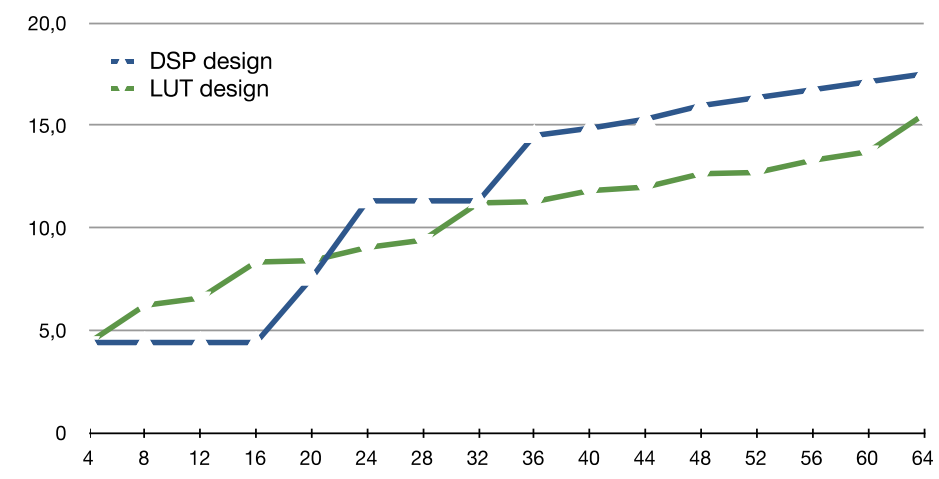

Figure 4 : Comparaison des chemins critiques (nanosecondes) entre une implantation à l'aide de logique câblée versus l'utilisation de blocs matériels précablés en fonction de la dynamique des calculs.

Cette conclusion associée à l'utilisation des blocs « optimisés » et de leurs piètres performances dans certains cas d'utilisation permet aux étudiants de prendre conscience de la nécessité de maitriser les performances des ressources employées lorsque l'on réalise un circuit matériel.

\section{Partie 3 : exemple d'opérateur « inefficace » : la division}

La dernière opération arithmétique que les étudiants doivent analyser au cours de cette séance est la division. Les divisions sont des opérations couramment utilisées en traitement du signal. Toutefois leur implantation matérielle est complexe: soit elle possède une latence élevée (architecture séquentielle), soit un coût prohibitif (architecture pipeline).

Il s'agit dans le cas présent d'évaluer les performances d'une division entière dont les opérandes sont codées sur 8 bits. Dans cette partie, les étudiants doivent écrire le code VHDL correspondant à la division dans sa forme combinatoire. Cette description est validée fonctionnellement à l'aide d'un testbench et d'une simulation à l'aide de l'outil ModelSim. Une synthèse à l'aide d'ISE permet d'obtenir les résultats d'implantation. Ces résultats sont étoffés à l'aide d'une description VHDL 
générique du diviseur fournie par l'enseignant. Les résultats obtenus par les étudiants sont fournis dans la Figure 5.
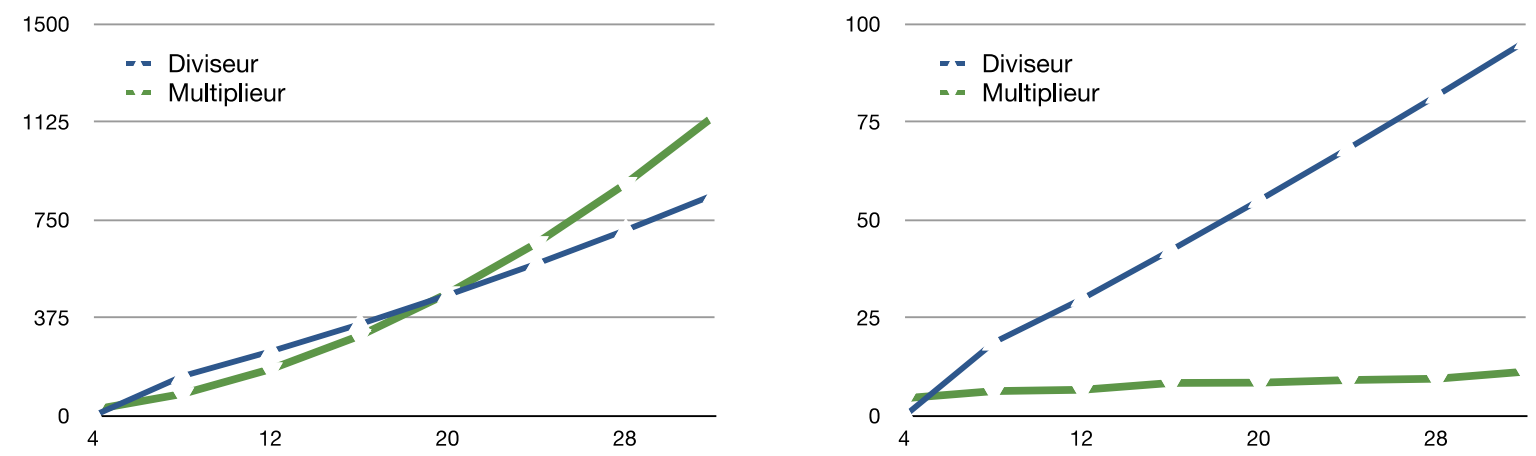

Figure 5 : Comparaison des performances obtenues lors de l'implantation d'un opérateur de division entière versus une multiplication (a) occupation en nombre de LUTs (b) délai du chemin critique en nanoseconde.

Cette série d'expériences démontre que le choix des opérations - réalisé lors de la définition des algorithmes à implanter peut impacter très fortement sur les performances matérielles attendues. Ainsi, le remplacement d'une division par d'autres types d'opérations peut améliorer grandement les caractéristiques du circuit à concevoir.

\section{Étape 2 : Étude des ressources de calcul flottantes (norme IEEE-745)}

Dans les phases d'études algorithmiques, généralement menées à l'aide de langage de haut niveau tel MATLAB, les données manipulées sont souvent au format flottant. Ce type de données fournit une précision suffisante pour bon nombre d'applications sans pour autant nécessiter un travail de la part du concepteur (étude de la dynamique des données). L'objectif du $2^{\text {ème }}$ TP est d'analyser les performances des opérateurs arithmétiques flottants une fois implantés sur FPGA.

\section{Partie 1 : étude de la multiplication flottante}

L'écriture d'un modèle VHDL synthétisable réalisant une multiplication flottante est une tâche complexe, inatteignable dans le cadre d'une séance de 3 heures. Pour cette raison, l'opérateur matériel est fourni aux étudiants.

La première tâche confiée aux étudiants est de retrouver l'algorithme réalisant la multiplication flottante à partir du code VHDL de l'opérateur. Le schéma bloc couramment fourni dans leur compte rendu est donné en Figure 6. 


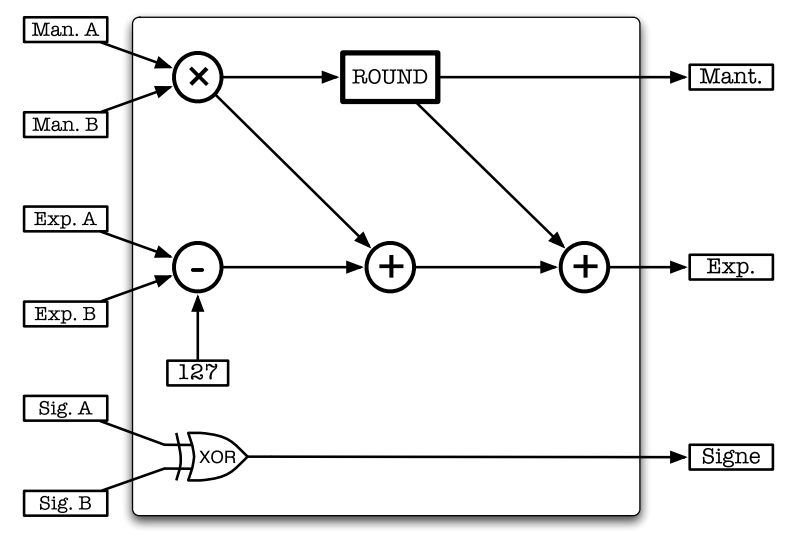

Figure 6: Schéma bloc décrivant le processus de multiplication de deux nombres flottants.

Cette analyse de l'opération de multiplication permet aux étudiants de comprendre que contrairement aux nombres codés en complément à 2 , les opérations sur les nombres flottants sont plus complexes à réaliser (algorithmiquement et matériellement). L'opérateur VHDL qui leur est fourni, est validé fonctionnellement à l'aide d'un testbench (conçu par les étudiants). Afin de les aider, un outil informatique réalisant la conversion entre nombre réel et codage 32 bits du nombre flottant norme IEEE-754 est mis à leur disposition.

L'étude de la multiplication flottante se conclut par une estimation des performances de l'opérateur après synthèse logique. Les résultats obtenus sont fournis dans le Tableau 1.

\begin{tabular}{l|c|c|c|c|c|c|c}
\hline & \# Slices & \# LUTs & $\begin{array}{c}\text { \# Flip- } \\
\text { Flop }\end{array}$ & $\begin{array}{c}\text { \# DSP } \\
\text { blocs }\end{array}$ & $\begin{array}{c}\text { Chemin } \\
\text { critique (ns) }\end{array}$ & $\begin{array}{c}\text { Débit (10^9 } \\
\text { ech./sec) }\end{array}$ & $\begin{array}{c}\text { Latence } \\
\text { (ns) }\end{array}$ \\
\hline Avec DSP & 57 & 103 & 0 & 4 & 16,837 & 59,39 & 16,837 \\
\hline Sans DSP & 372 & 734 & 0 & 0 & 14,301 & 69,93 & 14,301 \\
\hline
\end{tabular}

Tableau 1 : Résultats post-synthèse pour l'opérateur de multiplication (norme IEEE-754).

La surface occupée par l'opérateur de multiplication flottant est inférieure à celle obtenue pour la multiplication de nombres entiers codés sur 32 bits. Ce constat s'explique par la taille inférieure du multiplieur (24 bits) dans la multiplication flottante. Cependant le chemin critique de la multiplication flottante est plus long. Cette particularité est due à la propagation de la retenue entre le calcul de la mantisse et celui de l'exposant.

\section{Partie 2 : étude de l'addition flottante}

Afin de compléter l'estimation des performances des opérations flottantes vis-à-vis de leurs homologues entières, l'addition flottante est ensuite considérée. L'opérateur matériel d'addition est fourni sous forme VHDL synthétisable aux étudiants. Comme pour la multiplication flottante, les étudiants doivent analyser le processus d'addition en flottant afin de comprendre les calculs mis en œuvre. L'algorithme permettant d'additionner deux nombres flottant est bien plus complexe que celui réalisant leur multiplication. Cette complexité est due à l'alignement et au recadrage des mantisses. 
Suite à cette analyse, les étudiants réalisent la synthèse logique de l'opérateur afin d'estimer ses performances. Les résultats sont résumés dans le Tableau 2.

\begin{tabular}{l|c|c|c|c|c|c|c}
\hline & \# Slices & \# LUTs & $\begin{array}{c}\text { \# Flip- } \\
\text { Flop }\end{array}$ & $\begin{array}{c}\text { \# DSP } \\
\text { blocs }\end{array}$ & $\begin{array}{c}\text { Chemin } \\
\text { critique (ns) }\end{array}$ & $\begin{array}{c}\text { Débit (10^9 } \\
\text { ech./sec) }\end{array}$ & $\begin{array}{c}\text { Latence } \\
\text { (ns) }\end{array}$ \\
\hline Avec DSP & --- & --- & --- & --- & --- & --- & -- \\
\hline Sans DSP & 304 & 543 & 0 & 0 & 27,098 & 36,90 & 27,098 \\
\hline
\end{tabular}

Tableau 2 : Résultats post-synthèse pour l'opérateur d'addition (norme IEEE-754).

Ces résultats démontrent que l'implantation matérielle d'une addition flottante est moins performante que celle de la multiplication (surface et débit).

Dans le cas des opérateurs flottants, le coût d'une addition est bien supérieur (surface, délai) à celui d'une multiplication. Ce constat est à l'inverse de la conclusion réalisée lors de l'étude des opérations en complément à 2 .

\section{Partie 3 : utilisation de l'outil Core Generator de Xilinx}

Dans les étapes précédentes, notre étude s'est limitée à l'implantation de ressources de calcul combinatoires. Toutefois, il est possible de pipeliner les opérateurs flottants afin d'améliorer leurs performances (débit). Le découpage des chemins critiques est une étape complexe nécessitant une connaissance précise du processus de calcul. Pour cette raison, nous avons décidé d'utiliser l'outil Core Generator afin de générer de telles ressources.

Le travail des étudiants consiste dans cette partie à étudier les différents compromis offerts par l'outil Core Generator lors de la création de blocs matériels. Les étudiants règlent de manière visuelle les paramètres des opérateurs flottants (nombre de tranches de pipeline, taille des opérandes, ressources à employer) et observent instantanément l'estimation des performances (surface, fréquence maximum). Une capture d'écran de l'interface utilisée est fournie dans la figure 7. 


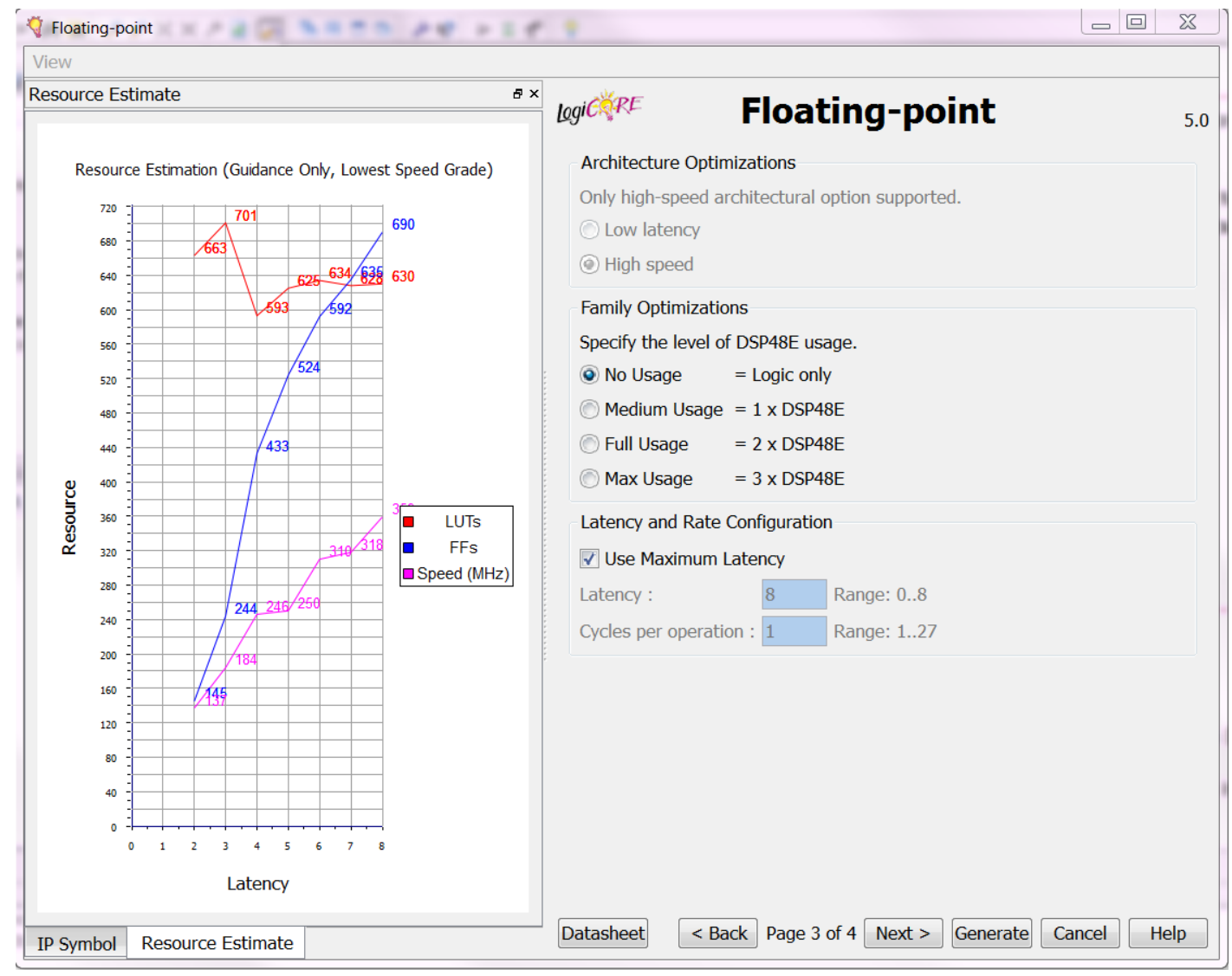

Figure 7 : Interface de paramétrage des opérateurs flottants dans l'outil CoreGenerator de Xilinx.

Cette partie permet de statuer sur l'impact des calculs flottants en termes de performances vis-à-vis de l'utilisation de ressources entières et la nécessité d'utiliser un codage des données en virgule fixe.

\section{Étape 3 : Conception d'accélérateurs matériels (filtres de type RIF)}

L'étude des opérateurs arithmétiques élémentaires a permis aux étudiants de comprendre l'impact de leurs décisions au niveau algorithmique sur les performances des accélérateurs matériels. Toutefois, l'étude ne portait que sur les opérateurs arithmétiques. Afin d'approfondir l'étude, nous avons décidé de réaliser un accélérateur matériel pour une application de traitement vidéo. Le composant de filtrage mis en œuvre dans ce TP est issu de la norme de compression d'image JPEG 2000 [Lawsonand 2002] [Jin 2003]. Nous allons mettre en œuvre le filtre RIF passe haut d'ordre 9. L'équation du filtre est fournie dans l'équation 1.

$$
\begin{aligned}
& Y(n)=0,02748757411 \quad X(n) \quad 0,016864118443 \quad X(n \quad 1) \quad 0,078223266529 \quad X\left(\begin{array}{ll}
n & 2
\end{array}\right) \\
& +0,266864118443 \quad X(n \quad 3)+0,602949018239 \quad X(n \quad 4)+0,266864118443 \quad X(n \quad 5) \\
& 0,078223266529 \quad X(n \quad 6) \quad 0,016864118443 \quad X(n \quad 7)+0,02748757411 \quad X(n \quad 8)
\end{aligned}
$$

Cette réalisation va mettre en exergue les différentes performances obtenues en fonction : du type de données manipulées et de l'architecture matérielle choisie pour implanter le filtre RIF. 


\section{Partie 1 : Implantation en virgule flottante du filtre passe-haut}

Dans un premier temps, les étudiants conçoivent l'accélérateur matériel correspondant à la projection matérielle directe de l'équation du filtre. Le circuit, proposé dans le sujet du TP correspond à une implantation combinatoire du filtre (Figure 8). Cette architecture est constituée d'une file de registres pour les échantillons et d'un registre permettant le maintien de la sortie. Afin d'implanter ce filtre, les étudiants doivent écrire le code VHDL permettant d'instancier les opérateurs flottants vus dans le TP précédent. La partie dédiée au vieillissement des échantillons est fournie dans un fichier VHDL.

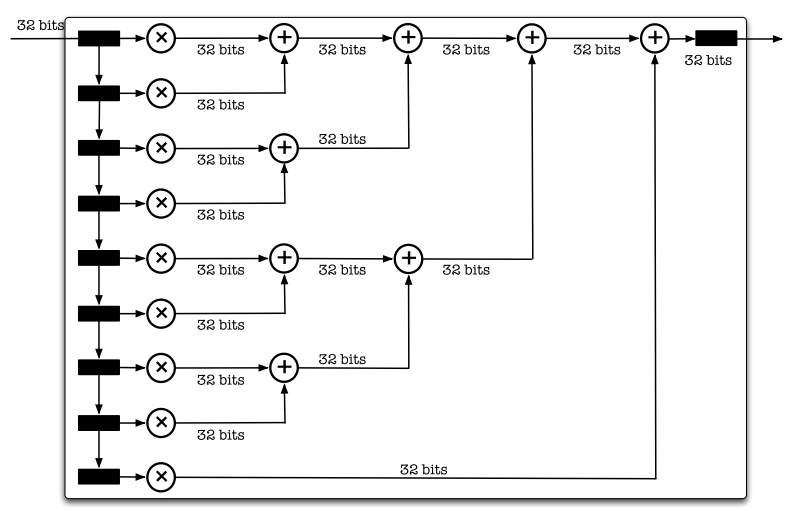

Figure 8 : structure du filtre passe-haut, implantation flottante et combinatoire

Suite à l'écriture du filtre passe-haut, dans sa déclinaison flottante, les étudiants valident son fonctionnement à l'aide d'un banc de test. Ce point, qui semble trivial, demande toutefois une certaine réflexion sur la valeur des échantillons à injecter dans le filtre afin de valider le circuit. Ce point est indispensable car la conversion des entrées et des sorties du filtre dans un format «lisible» est fastidieuse. En fonction de l'autonomie et de l'avancement des étudiants, soit ils développent leur propre solution de test, soit ils utilisent les deux approches de test proposées :

1. La première consiste à observer la réponse impulsionnelle du circuit. Si la valeur de l'impulsion est fixée arbitrairement à 1000, et que sa durée est d'un cycle d'horloge, alors on doit observer en sortie du filtre les valeurs des coefficients multipliées par un facteur 1000 avant que la sortie ne converge vers 0. Cette approche est schématisée en Figure 9.

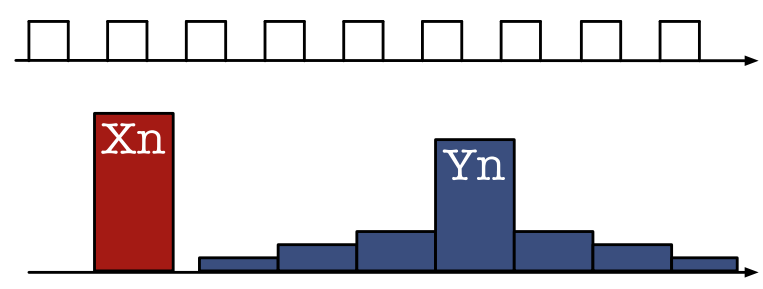

Figure 9 : Vérification fonctionnelle de la sortie (Yn) du filtre RIF à l'aide d'une impulsion en entrée (Xn)

2. La seconde consiste à observer la réponse indicielle du filtre. Si la valeur de l'échelon est fixée à 1.0 , et que sa durée est de 100 cycles d'horloge, alors on doit observer en sortie du filtre une 
valeur égale à la somme des coefficients du filtre, convergeant ainsi vers la somme totale des coefficients. Cette approche est schématisée en Figure 10.

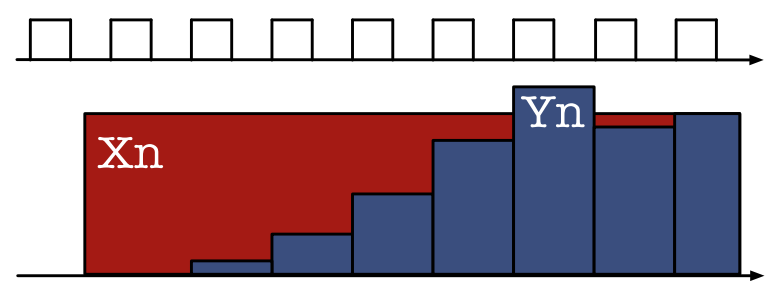

Figure 10 : Vérification fonctionnelle de la sortie (Yn) du filtre RIF à l'aide d'un échelon en entrée (Xn)

Une fois la fonctionnalité du filtre vérifiée, les étudiants réalisent sa synthèse logique afin d'évaluer le débit maximum et la surface occupée. Les résultats obtenus sont fournis dans le Tableau 3.

\begin{tabular}{l|c|c|c|c|c|c|c}
\hline & \# Slices & \# LUTs & $\begin{array}{c}\text { \# Flip- } \\
\text { Flop }\end{array}$ & $\begin{array}{c}\text { \# DSP } \\
\text { blocs }\end{array}$ & $\begin{array}{c}\text { Chemin } \\
\text { critique (ns) }\end{array}$ & $\begin{array}{c}\text { Débit (10^6 } \\
\text { ech./sec) }\end{array}$ & $\begin{array}{c}\text { Latence } \\
\text { (ns) }\end{array}$ \\
\hline Avec DSP & 3822 & 7417 & 320 & 36 & 93,810 & 10,66 & 187,62 \\
\hline Sans DSP & 6316 & 11633 & 320 & 0 & 92,031 & 10,87 & 184,062 \\
\hline
\end{tabular}

Tableau 3 : performances du filtre passe-haut, implantation flottante et combinatoire

Le débit du filtre est assez «faible» à cause de la longueur importante du chemin critique. Afin d'améliorer les caractéristiques en termes de débit de l'accélérateur matériel il est nécessaire d'introduire de nouveaux registres afin de «pipeliner » le filtre.

Les étudiants modifient alors l'architecture précédente en y ajoutant des registres entre les opérateurs arithmétiques. Cette étape a pour objectif de casser le chemin critique dans le circuit et donc d'en améliorer le débit. Le schéma du filtre obtenu après insertion des registres est fourni dans la figure 11.

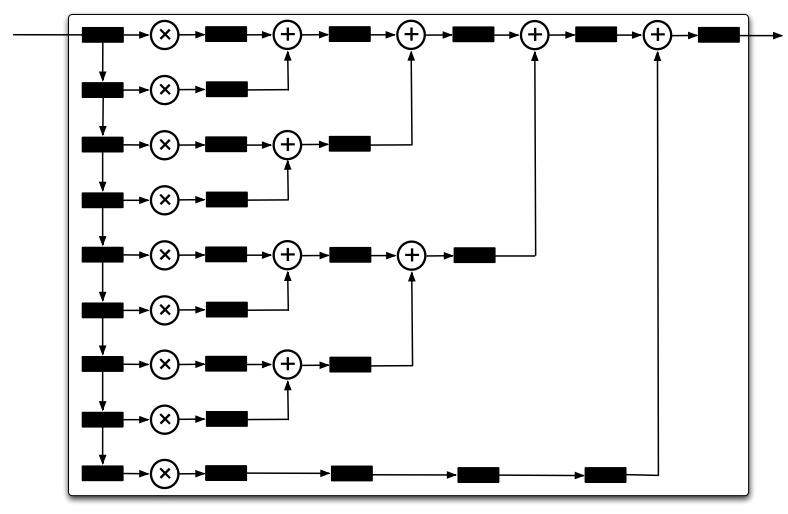

Figure 11 : Schéma bloc de l'implantation du filtre de type RIF dans sa forme pipeline (IEEE-754)

Une fois l'architecture réalisée, les étudiants vérifient qu'ils n'ont pas altéré le fonctionnement du circuit. Ils sont encouragés à reprendre le banc de test précédemment écrit (cela permet de vérifier sa non-régression). Cette étape franchie, une nouvelle évaluation des performances du filtre est réalisée à l'aide d'ISE. Les résultats obtenus sont indiqués dans le tableau 4. 


\begin{tabular}{l|c|c|c|c|c|c|c}
\hline & \# Slices & \# LUTs & $\begin{array}{c}\text { \# Flip- } \\
\text { Flop }\end{array}$ & $\begin{array}{c}\text { \# DSP } \\
\text { blocs }\end{array}$ & $\begin{array}{c}\text { Chemin } \\
\text { critique (ns) }\end{array}$ & $\begin{array}{c}\text { Débit (10^6 } \\
\text { ech./sec) }\end{array}$ & $\begin{array}{c}\text { Latence } \\
\text { (ns) }\end{array}$ \\
\hline Avec DSP & 3290 & 6362 & 825 & 36 & 21,276 & 47,00 & 127,656 \\
\hline Sans DSP & 5889 & 10799 & 825 & 0 & 21,108 & 47,38 & 126,648 \\
\hline
\end{tabular}

Tableau 4 : performances du filtre passe-haut, implantation flottante et pipeline

Ces résultats démontrent que l'architecture pipeline fournit un débit plus élevé que l'implantation combinatoire. Toutefois, cette architecture possède deux inconvénients : elle est plus coûteuse en surface et sa latence est plus importante.

Dans la partie suivante, nous nous attachons à améliorer les performances de l'architecture en optimisant le codage des données (virgule fixe). Cette étape nous permet de supprimer les opérateurs flottants qui constituent le goulot d'étranglement en termes de performances.

\section{Partie 2: Implantation en virgule fixe du filtre passe-haut}

Les implantations flottantes sont peu employées lors de la réalisation de circuits matériels. Afin d'améliorer les caractéristiques des circuits, un codage en virgule fixe des données est souvent préféré.

Le travail demandé aux étudiants consiste à réécrire les filtres flottants à l'aide d'opérations en virgule fixe. L'étude de l'impact du format de codage des données sur le bruit de calcul n'est pas réalisée dans ce TP, ce point étant abordé dans un autre enseignement. Les étudiants choisissent librement une taille comprise entre 12 et 24 bits pour les coefficients. Suite à ce choix et en amont du codage VHDL du filtre, les étudiants étudient la propagation de la dynamique des données dans les ressources matérielles. La figure 12 fournit ces résultats pour un codage sur 16 bits des coefficients. Une troncature est effectuée en sortie du filtre afin de supprimer la partie fractionnaire du résultat.

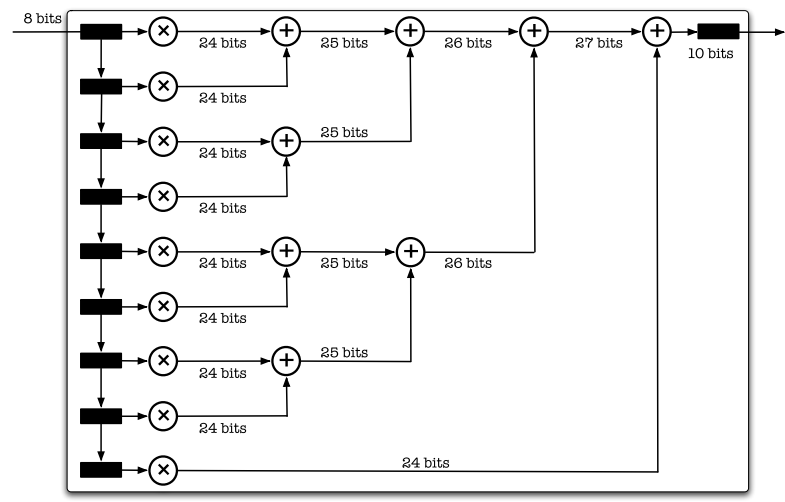

Figure 12 : architecture du filtre RIF dans sa projection combinatoire, annotée avec les largeurs nécessaires

Suite à cette étude théorique du filtre, les étudiants doivent compléter le fichier VHDL fourni. L'approche de conception employée pour les filtres flottants était de type structurel (PORT MAP). Ici les étudiants sont encouragés à utiliser une approche plutôt comportementale afin de simplifier l'architecture des filtres. La conversion des constantes flottantes en données codées en virgule fixe 
cadrées à gauche est réalisée simplement en utilisant à bon escient les capacités du langage VHDL (correction du filtre).

Suite à l'écriture des deux architectures VHDL, une étape de vérification fonctionnelle est à nouveau opérée. Les bancs de test utilisés lors de l'implantation des nombres flottants sont adaptés. Les étudiants terminent par la synthèse logique des filtres. Les résultats obtenus pour les implantations combinatoires et pipelines sont fournis respectivement dans les Tableaux 5 et 6 .

\begin{tabular}{l|c|c|c|c|c|c|c}
\hline & \# Slices & \# LUTs & $\begin{array}{c}\text { \# Flip- } \\
\text { Flop }\end{array}$ & $\begin{array}{c}\text { \# DSP } \\
\text { blocs }\end{array}$ & $\begin{array}{c}\text { Chemin } \\
\text { critique (ns) }\end{array}$ & $\begin{array}{c}\text { Débit (10^6 } \\
\text { ech./sec) }\end{array}$ & $\begin{array}{c}\text { Latence } \\
\text { (ns) }\end{array}$ \\
\hline Avec DSP & 92 & 175 & 32 & 8 & 11,992 & 83,39 & 23,984 \\
\hline Sans DSP & 498 & 834 & 80 & 0 & 12,603 & 79,35 & 25,206 \\
\hline
\end{tabular}

Tableau 5 : performances du filtre passe-haut, implantation combinatoire et virgule fixe

Le débit du filtre combinatoire dans sa version virgule fixe est bien supérieur à sa déclinaison en flottant. Le facteur d'accélération est ici de 8,33. Ce gain est à nuancer à cause de la précision employée pour coder les coefficients du filtre (ici 16 bits).

\begin{tabular}{l|c|c|c|c|c|c|c}
\hline & \# Slices & \# LUTs & $\begin{array}{c}\text { \# Flip- } \\
\text { Flop }\end{array}$ & $\begin{array}{c}\text { \# DSP } \\
\text { blocs }\end{array}$ & $\begin{array}{c}\text { Chemin } \\
\text { critique (ns) }\end{array}$ & $\begin{array}{c}\text { Débit (10^6 } \\
\text { ech./sec) }\end{array}$ & $\begin{array}{c}\text { Latence } \\
\text { (ns) }\end{array}$ \\
\hline Avec DSP & 53 & 89 & 82 & 11 & 3,591 & 278,47 & 21,546 \\
\hline Sans DSP & 517 & 853 & 468 & 0 & 6,854 & 145,90 & 41,124 \\
\hline
\end{tabular}

Tableau 6 : performances du filtre passe-haut, implantation pipeline et virgule fixe

Le débit du filtre RIF pipeline manipulant des données en virgule fixe s'avère être 5,9 fois supérieur à l'implantation flottante et 3,34 fois supérieur à l'implantation combinatoire et virgule fixe.

Afin de compléter l'évaluation des performances des architectures permettant d'implanter un filtre de type RIF d'ordre 9, les étudiants finissent par intégrer une architecture séquentielle. Cette architecture matérielle leur est fournie, directement codée en VHDL. Le travail des étudiants consiste à dimensionner le chemin de données qui est composé d'un seul opérateur de type MAC (Mutiply and ACcumulate). La Figure 13 procure une vue de l'architecture matérielle du filtre séquentiel.

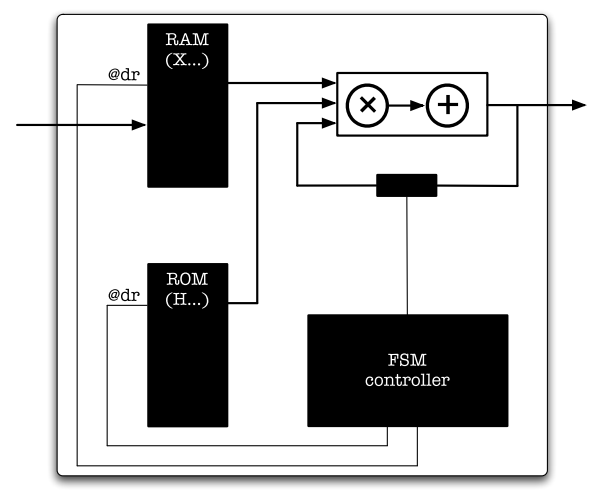

Figure 13 : Architecture matérielle du filtre RIF d'ordre 9 implanté dans sa forme séquentielle 
Pour une meilleure compréhension du fonctionnement du circuit, les étudiants doivent valider fonctionnellement le filtre fréquentiel. Suite à cette opération, la synthèse logique du circuit est réalisée afin de calculer les performances maximales de ce type de circuit.

\begin{tabular}{l|c|c|c|c|c|c|c}
\hline & \# Slices & \# LUTs & $\begin{array}{c}\text { \# Flip- } \\
\text { Flop }\end{array}$ & $\begin{array}{c}\text { \# DSP } \\
\text { blocs }\end{array}$ & $\begin{array}{c}\text { Chemin } \\
\text { critique (ns) }\end{array}$ & $\begin{array}{c}\text { Débit (10^6 } \\
\text { ech./sec) }\end{array}$ & $\begin{array}{c}\text { Latence } \\
\text { (ns) }\end{array}$ \\
\hline Avec DSP & 69 & 74 & 90 & 1 & 8,736 & 11,45 & 87,36 \\
\hline Sans DSP & 162 & 249 & 116 & 0 & 11,141 & 8,98 & 111,41 \\
\hline
\end{tabular}

Tableau 7 : performances du filtre passe-haut, implantation séquentielle en virgule fixe

Ce dernier type d'architecture, économique en surface vis-à-vis des autres approches possède néanmoins des performances en retrait (débit). Ce point s'explique par la présence d'une seule ressource de calcul. Il faut 10 cycles d'horloge afin de traiter un unique échantillon d'entrée.

Une comparaison des performances obtenues par les circuits conçus est fournie dans les Figures 14 et 15. Ces figures comparent respectivement les performances en terme de surface et de débit.

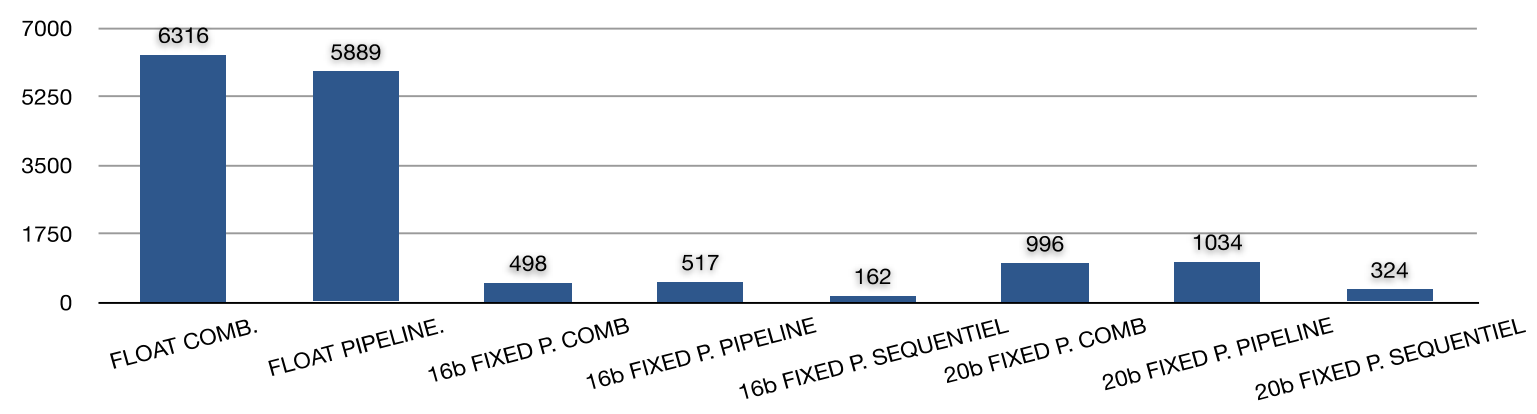

Figure 14 : XILINX Virtex-4 - Filtre RIF d'ordre 9 - Occupation du FPGA (nombre de slices)

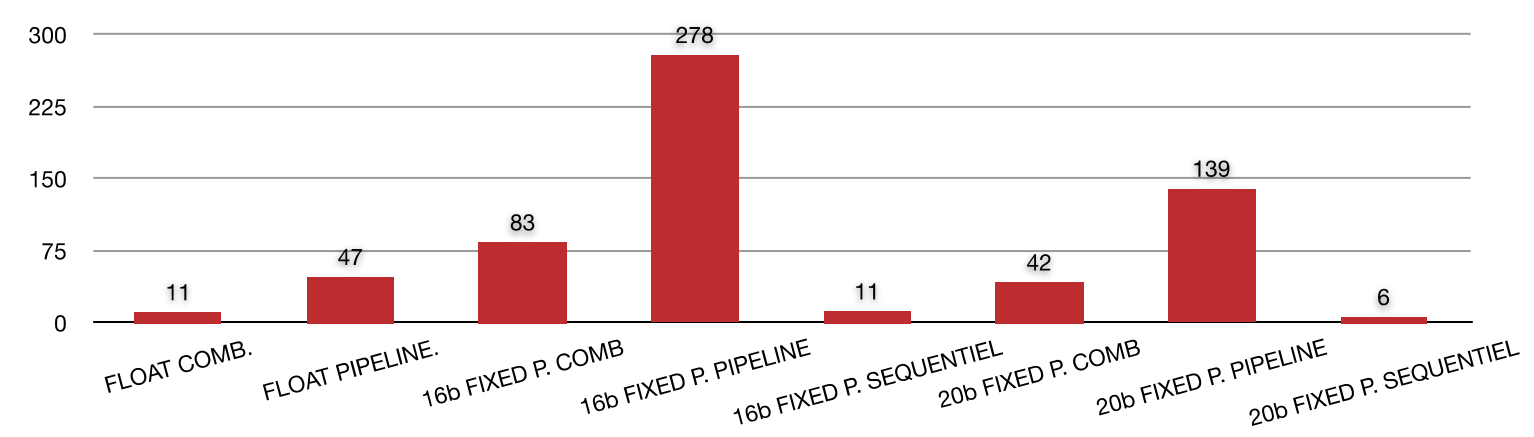

Figure 15 : XILINX Virtex-4 - Filtre RIF d'ordre 9 - Débit du filtre (10 échantillons par seconde)

\section{Partie 3 : Validation et estimation des gains en performances}

L'ensemble des résultats obtenus lors de la comparaison des architectures matérielles permet aux étudiants de comparer l'impact de l'architecture sur les performances du système. Toutefois, une réaction assez classique est leur « déception » vis-à-vis des fréquences de fonctionnement obtenues : « le filtre RIF flottant combinatoire ne peut fonctionner qu'à une fréquence de $10 \mathrm{MHz}=>$ le débit est limité à 10MS/s)». 
Les performances du filtre flottant dans sa forme combinatoire sont les plus faibles des architectures testées. Ces faibles performances sont à mettre en relief face aux performances que peut atteindre un processeur généraliste actuel (General Purpose Processor ou Digital Signal Processor). Afin de permettre aux étudiants de les comparer aux performances d'un processeur généraliste, un programme en langage $\mathrm{C}$ a été développé. Ce programme utilise une implantation classique du Filtre RIF d'ordre 9 dans quatre formats différents : double, float, virgule fixe (int) et optimisé en SSE [Intel 2002].

Le travail demandé aux étudiants consiste simplement à exécuter le programme fourni et à noter les résultats retournés. Le tableau 8 résume les résultats ${ }^{1}$ obtenus sur un ordinateur équipé d'un processeur Intel Core 2 Duo cadencé à 2.2GHz et possédant 2Go de mémoire RAM de type DDR3 à 1067GHz.

\begin{tabular}{c|c|c|c|c|c}
\hline & \multicolumn{2}{|c|}{ DOUBLE } & \multicolumn{2}{c|}{ FLOAT } & FIXED POINT (32b) \\
& C & SSE & C & SSE & C \\
\hline Temps (ms) & 18,1 & 11,9 & 16,5 & 10,2 & 9,9 \\
\hline Débit (Mb/s) & 55,148 & 84,376 & 60,752 & 98,418 & 100,760 \\
\hline
\end{tabular}

Tableau 7 : performances du filtre passe-haut, implantation sur un processeur généraliste (Intel Core 2 Duo)

A l'aide de ces résultats, il devient possible de comparer les performances en terme de débit entre ces deux types de solutions fonctionnant à des fréquences si différentes. Une synthèse des résultats obtenus est fournie dans la figure 16. Si nous nous attardons sur le cas du filtre flottant dans sa déclinaison pipeline, il est intéressant de remarquer que la fréquence de fonctionnement maximum est de $50 \mathrm{MHz}$ comparée à celle du processeur à $2200 \mathrm{MHz}$. Ce rapport 44 pouvait (pour les étudiants) présager d'un débit nettement inférieur pour le FPGA, dans le cas présent, le débit de la solution sur FPGA est seulement 20\% inférieur. A l'inverse, l'implantation sur FPGA du filtre en virgule fixe avec une architecture pipeline fonctionnant à $278 \mathrm{MHz}$, atteint des débits 2,8 fois plus importants que la solution logicielle.

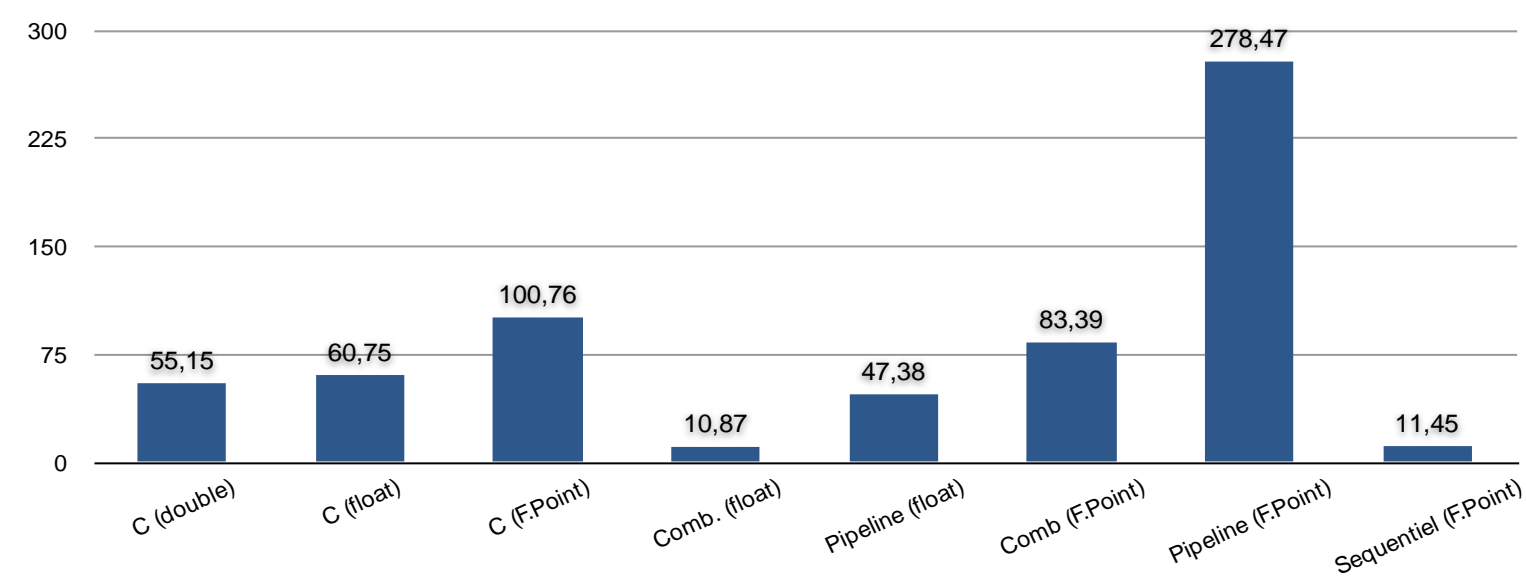

Figure 16: comparaison des approches logicielles et matérielle en fonction du débit maximum obtenu (résultats exprimés en kilo-échantillons par seconde, échelle logarithmique).

\footnotetext{
${ }^{1}$ Le temps indiqué dans le tableau correspond à l'exécution du filtre RIF sur $10^{6}$ échantillons.
} 
L'objectif pédagogique atteint à la fin de cette séquence pédagogique est triple. Les étudiants ont compris :

- l'impact de l'agencement des ressources matérielles sur les performances,

- l'impact du codage en virgule fixe sur : la taille des ressources à utiliser, les performances globales des architectures matérielles et sur la complexité de mise en œuvre qui a été appréhendée et évaluée,

- finalement les résultats issus de la comparaison avec un processeur généraliste bien plus rapide (fréquence de fonctionnement), programmé en C, justifie l'intérêt des accélérateurs matériels.

\section{Étape 4 : Mise en ouvre sur carte}

L'objectif des séances de TP précédentes était d'évaluer les performances théoriques que l'on pouvait obtenir lors de l'implantation d'un filtre de type RIF sur FPGA. Ces gains sont difficilement atteignables lorsque l'on ne conçoit pas intégralement le système (alimentation du filtre à de très hauts débits). Afin de valider le bon fonctionnement pratique du filtre déjà conçu, au sein du FPGA, nous avons décidé de coupler l'accélérateur matériel à un processeur de type SoftCore.

La manipulation mise en œuvre emploie un processeur Microblaze [Xilinx 2008c] conçu et distribué par Xilinx. Son objectif principal sera d'alimenter en données l'accélérateur matériel, de récupérer les résultats des calculs et de valider les résultats obtenus par rapport à ceux délivrés par l'implantation $\mathrm{C}$ du filtre. Au sein du FPGA, le lien entre le processeur et l'accélérateur matériel est réalisé de 2 manières différentes :

- liaison multipoints à travers un bus périphérique (Processor Local Bus, PLB [Xilinx 2009]). En utilisant ce mode d'interconnexion, l'accélérateur matériel est relié au processeur à l'aide d'un bus partagé. Ce bus partagé est employé par d'autres ressources matérielles (mémoire, DMA, UART, lien Ethernet, etc.). Un schéma décrivant le système est fourni en figure 19a.

- liaison point à point à travers un Fast Simplex Link (FSL) [Xilinx 2010][Rosinger 2004]. Les liens FSL (Figure 19b) sont des canaux de communication privilégiés avec le processeur car ils sont dédiés. Toutefois, un processeur softcore ne possède qu'un nombre fini de canaux FSL.
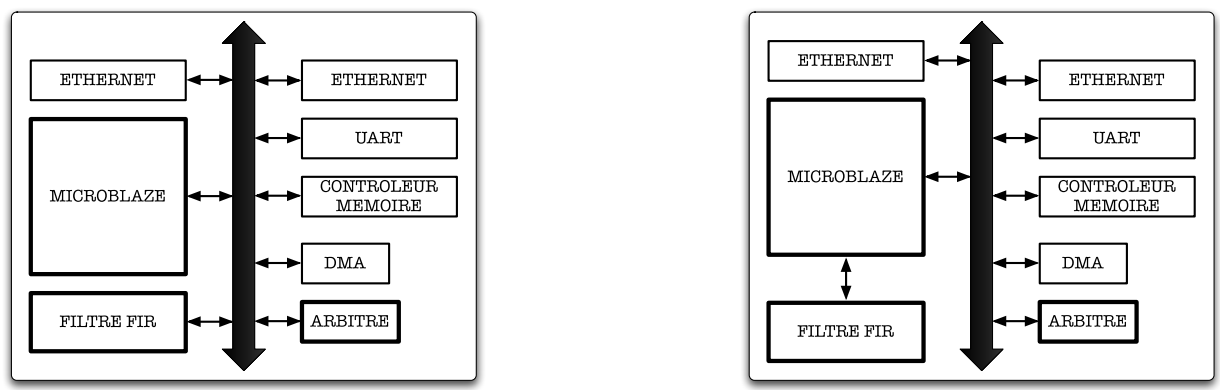

Figure 19 : Architecture système permettant de lier l'accélérateur matériel au processeur à l'aide
(a) d'un lien multipoints - bus $O P B$
(b) d'un lien point à point - lien FSL 
L'utilisation de ces deux mécanismes de communication accessibles lors de l'utilisation d'un Microblaze permet aux étudiants de comprendre le concept général: ils ajoutent l'accélérateur matériel comme un «simple » périphérique. De plus, elle met en exergue la baisse des performances imposée par le temps de communication entre le processeur et l'accélérateur matériel. Ce temps de communication va devenir le principal goulot d'étranglement pour le système.

A travers un tutorial présentant pas à pas les différentes étapes de configuration du système, les étudiants vont créer le circuit implantant le processeur SoftCore et leur accélérateur matériel. Suite à la phase de synthèse des parties matérielles et de compilation des parties logicielles, les étudiants vont valider fonctionnellement le design sur la carte.

Les temps de calcul mesurés et les débits atteints par le système en fonction des différentes configurations sont fournis dans le tableau 8.

\begin{tabular}{c|c|c|c|c} 
Implantation & $\begin{array}{c}\text { \# échantillons } \\
\text { par transfert }\end{array}$ & $\begin{array}{c}\text { Nombre de } \\
\text { cycles d'horloge }\end{array}$ & $\begin{array}{c}\text { \# cycles moyen } \\
\text { par données }\end{array}$ & Gain \\
\hline Software & ---- & 48028771 & 48,0 & ---- \\
\hline \multirow{2}{*}{ PLB } & 1 & 27024739 & 27,0 & $43,73 \%$ \\
\cline { 2 - 5 } & 4 & 6774758 & 6,8 & $85,89 \%$ \\
\hline \multirow{2}{*}{ FSL } & 1 & 11021668 & 11,0 & $77,05 \%$ \\
\cline { 2 - 5 } & 4 & 4270371 & 4,3 & $91,11 \%$ \\
\hline
\end{tabular}

Tableau 8 : performances du filtre passe-haut, implantation flottante et pipeline

Les mesures de performance ont été réalisées dans les conditions suivantes :

- La fréquence du processeur SoftCore a été fixée à $100 \mathrm{MHz}$,

- La fréquence de fonctionnement du bus PLB a été fixée à $100 \mathrm{MHz}$,

- La taille des FIFOs instanciées pour les liens FSL est de 64 données.

- Le vecteur de test est composé de un méga-échantillons (les données sont codées sur 8 bits).

Comme le démontre le tableau 8 , l'utilisation de l'accélérateur matériel permet de réduire de façon considérable le temps de traitement des échantillons vis-à-vis d'une implantation purement logicielle. Le temps de traitement du vecteur d'échantillons est réduit au minimum de $44 \%$ et les gains s'élèvent au maximum à $91 \%$.

Différents types de communication ont été évalués afin d'améliorer le débit. Tout d'abord on peut remarquer que les communications via un lien FSL sont plus performantes que celles réalisées à l'aide du bus PLB (qui est plus générique). La différence de performances s'explique par le fait que les liens de type FSL ne nécessitent pas d'étape d'arbitrage. Finalement, le débit maximum est obtenu lorsque nous utilisons de manière optimum la taille du bus de données : les données sont codées sur 8 bits alors qu'un transfert de données sur le bus permet de transmettre simultanément 32 bits. Afin d'exploiter cette spécificité de l'application, nous avons ajouté à coté du filtre un adaptateur qui (1) récupère 4 données simultanément (2) découpe les 32 bits sous forme de 4 paquets de 8 bits (3) réalise 
les 4 calculs à l'aide de l'accélérateur matériel (4) regroupe les résultats sous forme d'un mot de 32 bits.

En conclusion, cette dernière partie de la séquence pédagogique permet aux étudiants de se confronter à l'intégration de l'accélérateur matériel sur FPGA. De plus l'intégration se réalise dans un flot de conception conjoint.

\section{Bilan pédagogique}

La séquence pédagogique décrite dans cet article est maintenant enseignée depuis 3 ans. Malgré le fait que l'établissement organise chaque année des enquêtes qualité sur les enseignements prodigués, il est impossible d'utiliser ces informations. En effet le nombre d'étudiants répondant à l'enquête est excessivement faible, voir nul. Cependant des discussions directes avec les étudiants ainsi que les notes obtenues démontrent que cette sensibilisation intéresse les étudiants. Ce sentiment est renforcé par les questions pertinentes qui sont posées durant les séances de cours.

Toutefois, si l'on se place du point de vue de l'enseignant, afin de s'assurer une compréhension homogène des phénomènes observés, il est nécessaire d'encadrer activement les étudiants lors des phases d'analyse et de synthèse des résultats. En effet, dans le cas contraire, l'analyse des résultats est trop souvent superficielle. Cela implique de la part de l'enseignant un investissement important dans le suivi des étudiants lors de la rédaction des comptes-rendus.

\section{Conclusion}

Les différentes parties de ce TP permettent aux étudiants de développer leurs compétences et connaissances en conception de systèmes numériques et tout particulièrement dans le domaine des accélérateurs matériels. La séquence pédagogique dont le sens est inverse à celui de la conception traditionnelle, leurs fait comprendre l'impact à bas niveau des contraintes induites par les algorithmes qu'ils peuvent être amené à concevoir. L'intérêt des accélérateurs matériels et les problèmes liés à leur intégration sont illustrés et mis en œuvre autour d'un filtre issu de la norme JPEG-2000. Finalement, la comparaison des performances obtenues sur FPGA avec celles obtenues sur un processeur généraliste de type Pentium Core 2 Duo relativise l'impact réel de la fréquence de fonctionnement des circuits vis-à-vis de leurs architectures internes.

\section{Remerciements}

Les cartes et le logiciel ISE utilisé dans ce TP ont été fournis par le Xilinx University Program (http://www.xilinx.com/univ/) via la Coordination Nationale pour la Formation en Micro et nanoélectronique (http://www.cnfm.fr/). 


\section{Bibliographie}

[Ercegovac 2003] Milos Ercegovac et Tomas Lang, "Digital Arithmetic”. Morgan Kaufmann, ISBN: 155860-798-6, 2003.

[Intel 2002] Intel Corporation, "Intel ${ }^{\circledR} 64$ and IA-32 Architectures Optimization Reference Manual”. Reference: 248966-021, August 2010

[Jin 2003] Jin Li, “Image Compression: The Mathematics of JPEG 2000”. Modern Signal Processing, MSRI Publications, volume 46, page 185, 2003.

[Lawsonand 2002] S. Lawsonand and J. Zhu, "Image compression using wavelets and JPEG2000: a tutorial". Electronics and Communication Engineering Journal, page 112, June 2002.

[Rosinger 2004] Hans-Peter Rosinger, "Connecting Customized IP to the MicroBlaze Soft Processor Using the Fast Simplex Link (FSL) Channel”. reference XAPP529, revision 1.3, May 2004

[Tisserand 2004] Arnaud Tisserand, “Conception d'opérateurs arithmétiques: aspects matériels”. Séminaire CCA, ENSTA Paris, 12 mars 2004.

[Xilinx 2008a] Xilinx Corporation, “Virtex-4 FPGA User Guide”. reference UG070, revision 2.6, December 1, 2008.

[Xilinx 2008b] Xilinx Corporation, “XtremeDSP for Virtex-4 FPGAs - User Guide”. reference UG073, revision 2.7, May 2008.

[Xilinx 2008c] Xilinx Corporation, "MicroBlaze Processor Reference Guide Embedded Development Kit EDK 10.1”. revision 9.0, January 2008.

[Xilinx 2009] Xilinx Corporation, "Processor Local Bus (PLB) v4.6”. revision 1.04a, reference DS531, December 2009.

[Xilinx 2010] Xilinx Corporation, “LogiCORE IP Fast Simplex Link (FSL) v2.0 Bus”. revision 2.11c, reference DS449, April 2010

Bertrand Le Gal a obtenu un DESS en Mécatronique ainsi qu'un DEA en Électronique à l'université de Bretagne Sud en 2002 et le Doctorat en Sciences et Sciences de l'Ingénieur de l'Université de Bretagne Sud à Lorient en 2005. Il a effectué sa thèse au laboratoire LESTER à Lorient sur le thème de l'exploration de l'espace de conception des architectures reconfigurables. Après une année d'ATER à l'ENSSAT à Lannion, il a rejoint en 2006 en tant que Maître de Conférences le laboratoire IMS et l'Institut Polytechnique Bordeaux (IPB) de Bordeaux ou il enseigne principalement l'électronique numérique et plus particulièrement l'Adéquation Algorithme Architecture. Ses recherches portent sur l'Adéquation Algorithme Architecture appliquée aux systèmes multimédias, les méthodologies automatisées de conception et l'amélioration des performances des circuits analogiques et mixtes à l'aide de structures numériques.

Dominique Dallet est Professeur des Universités à l'Institut Polytechnique Bordeaux (IPB) depuis septembre 2004 et effectue ses activités de recherche au sein de l'IMS depuis septembre 1991. Il a 
obtenu son doctorat en janvier 1995 sur des travaux concernant la caractérisation dynamique des convertisseurs analogique-numérique. Il a étendu ses activités autour des systèmes de conversion de données, de la conception au test en passant par la modélisation. C'est sur ces activités qu'il a présenté son Habilitation à diriger la recherche (HDR) en décembre 2003. Actuellement, il est responsable de l'équipe 'Circuits et Systèmes Numériques' du laboratoire IMS et ses activités de recherche sont axées sur l'amélioration des performances des circuits RF et mixtes par des structures numériques (compensation PA, filtrage des architectures de CAN entrelacés). 